\title{
A STONE-WEIERSTRASS THEOREM FOR GROUP REPRESENTATIONS
}

\author{
JOE REPKA \\ Department of Mathematics \\ University of Toronto \\ Toronto, Ontario, Canada M5S 1A1 \\ (Received January 16, 1978)
}

ABSTRACT. It is well known that if $G$ is a compact group and $\pi$ a faithful (unitary) representation, then each irreducible representation of $G$ occurs in the tensor product of some number of copies of $\pi$ and its contragredient. We generalize this result to a separable type I locally compact group G as follows: let $\pi$ be a faithful unitary representation whose matrix coefficient functions vanish at infinity and satisfy an appropriate integrability condition. Then, up to isomorphism, the regular representation of $G$ is contained in the direct sum of all tensor products of finitely many copies of $\pi$ and its contragredient. We apply this result to a symplectic group and the Weil representation associated to a quadratic form. As the tensor products of such a representation are also Weil representations (associated to different forms), we see that any discrete series representation can be realized as a subrepresentation of a Well representation. 
1. INTRODUCTION.

Let $G$ be a separable locally compact group, and $\pi$ a representation of $G$ ("representation" will always mean a strongly continuous unitary representation on a separable Hilbert space). For every pair of non-negative integers $m, n$, not both zero, let

$$
\pi_{m, n}=(\pi \otimes \cdots \otimes \pi) \otimes(\bar{\pi} \otimes \cdots \otimes \bar{\pi}),
$$

where $\pi$ occurs $m$ times, and its contragredient $\bar{\pi}$ occurs $\mathrm{n}$ times.

our starting point is the following easy fact.

THEOREM 1. Let $G$ be a compact group and $\pi$ a faithful representation. Then every irreducible representation of $G$ occurs in

$$
\sigma=\underset{m, n \geq 0}{\oplus^{\prime}} \pi_{m, n},
$$

where the prime means we omit $(m, n)=(0,0)$ from the sum.

PROOF. Consider the set of linear combinations of the matrix coefficient functions of the representation $\sigma$. These functions form an algebra, are closed under complex confugation, separate points of $G$, and do not all vanish at any point of $G$. By the Stone-Weierstrass Theorem, they are supnorm dense in the continuous functions on $G$, and hence dense in $L^{2}(G)$. But, by the Peter-Weyl Theorem, a representation whose coefficient functions are dense in $L^{2}(G)$ must contain every irreducible representation.

REMARK. Since, as is well known, $\pi \otimes \bar{\pi}$ contains the trivial representtation, it is easy to see that every irreducible representation occurs not just once, but countably infinitely many times in $\sigma$.

This theorem, in a slightly different form, is originally due to Burnside, in the case of a finite group G (see, e.g., Curtis and Reiner [2], Theorem 
32.9); namely:

THEOREM 2. (Burnside). Let $G$ be a finite group, $\pi$ a faithful representation. Then every irreducible representation of $G$ occurs in the tensor product of some number of copies of $\pi$.

Rather than prove Burnside's theorem directly, we obtain it as an obvious special case of the following.

THEOREM 3. Let $\mathrm{n}$ be a positive integer. Let $\mathrm{G}$ be a compact group, all of whose elements have order dividing $n$. If $\pi$ is a faithful representation of $G$, then every irreducible representation of $G$ occurs in the tensor product of some number of copies of $\pi$.

PROOF. The argument is the same as that for Theorem 1; the only thing that is not obvious is that the algebra spanned by coefficient functions is closed under complex conjugation. But the complex conjugates of the matrix coefficients of $\pi(g)$ are just the matrix coefficients of $\pi(g)^{-1}$ rearranged (since $\pi$ is unitary), and the matrix coefficients of $\pi(g)^{-1}=\pi(g)^{n-1}$ are just sums of products of matrix coefficients of $\pi(g)$, so we are done.

We generalize Theorem I to a non-compact group.

\section{PRELIMINARIES.}

In this section we establish two results needed for the proof of the main theorem. As above, $\mathrm{G}$ is a separable locally compact group.

Let $\psi$ be a continuous square-integrable function on $G$. Let $U$ be the open set where $\psi$ is not zero. We may restrict Haar measure to $U$ and speak of the closed subspace $L^{2}(U) \subseteq L^{2}(G)$, consisting of functions which vanish off $U$. 
LEMMA 4. Let $A$ be an algebra of continuous functions on $G$ which vanish at infinity and separate points, and suppose $A$ is closed under complex conjugation. Let $\psi, U$ be as above.

Then $\psi \cdot A=\{\psi \cdot f: f \in A\}$ is dense in $L^{2}(U)$.

PROOF. Let $\phi \in \mathrm{C}_{\mathrm{c}}(\mathrm{U})$, and fix $\varepsilon>0$. Then $\phi / \psi \in \mathrm{C}_{\mathrm{C}}(\mathrm{U})$, and by the Stone-Weierstrass Theorem there exists $\phi^{\prime} \in A$ such that $\left\|\phi^{\prime}-\phi / \psi\right\|_{\infty}<\varepsilon$. Consequently, $\left\|\psi \cdot \phi^{\prime}-\phi\right\|_{2}=\left\|\psi\left(\phi^{\prime}-\phi / \psi\right)\right\|_{2} \leq\|\psi\|_{2} \cdot \varepsilon$, and we have approximated $\phi$ by a function in $\psi \cdot A$. Since $C_{c}(U)$ is dense in $L^{2}(U)$, the proof is complete.

Now let $\sigma$ be a representation of $G$ on a Hilbert space $H$.

LEMMA 5. Suppose the intersection of $L^{2}(G)$ with the set of coefficient functions of $\sigma$ spans a dense subspace of $L^{2}(G)$. Then (up to isomorphism) the regular representation of $G$ is quasi-contained in $\sigma$ (i.e. contained in a direct sum of copies of $\sigma$ ).

PROOF. If $u, v \in H$ are such that the coefficient function $\phi_{u, v}(g)$ $=\langle\sigma(g) u, v\rangle$ is in $L^{2}(G)$, we define a linear map $T_{v}$ from some subspace of $H$ to $L^{2}(G)$ by $T_{v}(w)=\phi_{w, v}$.

The domain of $T_{V}$ will be all vectors $w \in H$ such that $\phi_{W, v}$ is squareintegrable; it is a G-invariant subspace, containing $u$. It is easy to check that $T_{V}$ is a closed G-map; hence we may apply Schur's Lemma to conclude that there are closed G-subspaces $V_{V} \subseteq H, W_{V} \subseteq L^{2}(G)$ and a surjective unitary G-isomorphism $U_{v}: V_{v} \rightarrow W_{v}$. Moreover, $W_{v}$ contains the function $\phi_{u, v}$.

By hypothesis we may choose (countably many) coefficient functions $\phi_{u, v}$ whose span is dense in $L^{2}(G)$. The corresponding subspaces $W_{V}$ thus span a dense subspace of $L^{2}(G)$, and, sticking together the maps $U_{V}^{-1}$ and 
applying Schur's Lemma again, we get a unitary G-isomorphism of $L^{2}(G)$ into $\underset{v}{\oplus} V_{V} \subseteq \underset{V}{\oplus} H$, a countable direct sum of copies of $H$.

\section{THE MAIN RESULT.}

We shall prove two weaker versions of our main result as a preliminary step.

PROPOSITION 6. Let $G$ be a separable locally compact group, and $\pi$ a faithful representation of G. Suppose that a non-trivial coefficient function of $\pi$ is in $L^{p}(G)$, for some finite $p$, and that all the coefficient functions vanish at infinity.

Then the regular representation of $G$ is quasi-contained in

$$
\sigma=\oplus_{m, n \geq 0}^{\prime} \pi_{m, n}
$$

PROOF. By Lemma 5, it suffices to find coefficient functions of $\sigma$ whose span is dense in $L^{2}(G)$. Suppose $\phi$ is a coefficient function of $\pi$ which is in $L^{p}(G)$. Then some power $\phi^{n}$ of $\phi$ is in $L^{2}(G)$. Since $\phi^{n}$ is a coefficient function of $\pi_{n, 0}$, we see that the algebra $A$ spanned by the coefficient functions of $\sigma$ contains $\phi^{n} \cdot A$ (note $\sigma$ contains $\pi_{n, 0} \otimes \sigma$ ). By Lemma 4, $A \cap L^{2}(G)$ contains a dense set of $L^{2}(U)$, where $U=\{g \in G: \phi(g) \neq 0\}$.

Since $A \cap L^{2}(G)$ is invariant under translations by $G$, we see that it contains a dense subset of $L^{2}(g \cdot U)$, for any $g \in G$. But since $U$ is open, any element of $L^{2}(G)$ can be approximated by a finite sum of elements belonging to spaces $L^{2}(g \cdot U)$, for appropriate choices of $g \in G$. Such a sum can be approximated by something in $A \cap L^{2}(G)$.

We have approximated an arbitrary element of $L^{2}(G)$ by a sum of elements of $A \cap L^{2}(G)$, as desired. 
In fact, we can strengthen this result slightly, as follows:

PROPOSITION 7. Let $\pi, \sigma$ be as above, and let $\tau$ be any representation of G . Then the regular representation of $G$ is quasi-contained in $\tau \sigma$.

PROOF. The proof is analogous to that of Proposition 6; we just replace $\phi^{\mathrm{n}}$ by $\phi_{0} \cdot \phi^{\mathrm{n}}$, where $\phi_{0}$ is any coefficient function of $\tau$, and let $U=\left\{g \in G: \phi_{0} \cdot \phi(g) \neq 0\right\}$.

We are now ready to prove the main result, which is essentially Proposition 7 with "quasi-contained" changed to "contained".

THEOREM 8. Let $G$ be a separable type I locally compact group, $\pi$ a faithful representation whose coefficient functions vanish at infinity and one of whose non-trivial cofficient functions is in ${ }_{L}^{p}(G)$ for some finite $p$. Let $\tau$ be any representation.

Then the regular representation of $G$ is (up to unitary isomorphism) contained countably infinitely many times in $\tau \otimes \sigma=\tau \otimes\left(\begin{array}{cc}\oplus^{\prime} & \pi_{m, n} \\ m \geq 0\end{array}\right)$.

PROOF. We shall show that every subrepresentation of the regular representation occurs not just once but infinitely many times in $\tau \approx \sigma$. It will suffice to consider multiplicity-free subrepresentations, i.e. those of the form

$$
n=\int_{\hat{G}}^{\oplus} \nu d \mu(\nu)
$$

where $\hat{G}$ is the set of equivalence classes of unitary irreducible representations of $G$, and $\mu$ is some finite Radon measure on $\hat{G}$ which is absolutely continuous with respect to the Plancherel measure. Fix such a $\mu$, and fix $\varepsilon>0$. By Proposition 7, we know that $\eta$ is contained in $\tau \sigma$, so there 
is a positive integer $M(1)$ such that $\tau-\sigma_{1}=\tau \cdot\left(\int_{m, n<M(1)}^{\prime} \pi_{m, n}\right)$

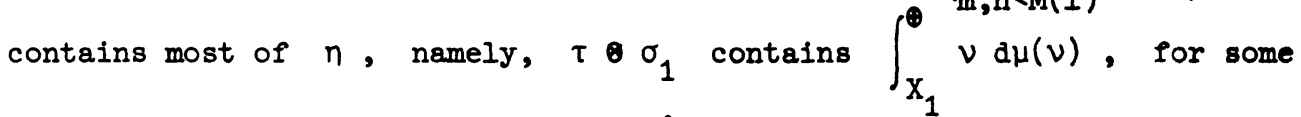
measurable set $X_{1} \subseteq \hat{G}$ such that $\mu\left(\hat{G} \backslash X_{1}\right)<\varepsilon / 2$.

Now consider the representation $\tau \otimes \pi_{M(I), M(1)} \otimes \sigma=\tau \otimes\left(\begin{array}{ll}\oplus_{M(I) \leq m, n}^{\prime} & \pi_{m, n}\end{array}\right)$ (the prime means omit $(m, n)=(M(1), M(1))$ ). By Proposition 4, this representation contains $\eta$, so we may find a measurable set $x_{2} \subseteq \hat{G}$ with $\mu\left(\hat{G} \backslash X_{2}\right)<\varepsilon / 4$ and a positive integer $M(2)$ such that $\tau \bullet \sigma_{2}$ $=\tau \theta\left(\begin{array}{cc}\oplus_{M(1) \leq m, n<M(2)}^{\prime} & \pi_{m, n}\end{array}\right)$ contains $\int_{X_{2}}^{\oplus} \nu \mathrm{d} \mu(\nu)$.

Continuing inductively, we find measurable sets $x_{1}, x_{2}, x_{3}, \ldots \subseteq \hat{G}$ such that $\mu\left(\hat{G} \backslash X_{k}\right)<2^{-k} \cdot \varepsilon$, and integers $M(1)<M(2)<M(3)<\ldots$ such that $\tau \otimes \sigma_{k}=\tau \cdot\left(\begin{array}{ll}\oplus_{M(k-1) \leq m, n<M(k)}^{\prime} & \pi_{m, n}\end{array}\right)$ contains $\int_{X_{k}}^{\oplus} \nu d \mu(\nu)$.

But $\sigma$ contains $\sigma_{1} \oplus \sigma_{2} \oplus \sigma_{3} \oplus \ldots$, so $\tau * \sigma$ contains countably infinitely many copies of $\int_{X}^{\oplus} \nu d \mu(\nu)$, where $x=n_{X_{k}}$. Now $\mu(\hat{G} \backslash X)<\varepsilon$, and since $\varepsilon$ was arbitrary, the theorem is proved.

\section{WEIL REPRESENTATIONS AND THE DISCRETE SERIES.}

The Weil representation has long been known as a fruitful place for realizing various irreducible representations (see, e.g., Gelbart, [3] and Howe, [5]). We shall apply Theorem 8 to show that any discrete series representation of a symplectic group can be so realized.

In (Weil, [7]) there is constructed a representation of the symplectic group associated to a quadratic form $Q$. The tensor products of this representation with itself and/or its contragredient are again Weil representations, those associated to forms constructed by taking direct sums of copies of $\pm Q$. Since the discrete series representations of $G$ are sub- 
representations of the regular representation, we have as an immediate consequence of Theorem 8:

THEOREM 9. With $G$ as above, any representation of the discrete series of $G$ is a subrepresentation of some Weil representation of $G$ (associated to some quadratic form).

\section{COMPLEMENTS.}

In this section we make some remarks and give some examples.

The conditions on $\pi$ - that its matrix coefficient functions must vanish at infinity, and that at least one of them must be in some $L^{p}(G)$ seem rather peculiar at first. We remark that they are necessary, for example, to exclude finite dimensional representations of a non-compact group (indeed, if $\pi$ were finite dimensional, then $\sigma$ would be a direct sum of finite dimensional representations, and could not possibly contain the regular representation). On the other hand, though something of this sort is needed, the conditions we have given can be altered or weakened in various ways; we give two examples. Firstly, it is not necessary to know that the coefficient functions all vanish at infinity. It would suffice to know that the ones belonging to some dense set of vectors did so (e.g. $\mathrm{K}$-finite vectors), or even just that one coefficient function achieves its maximum absolute value on a compact set. Suitable modifications of Lemma 4 hold in these cases. Secondly, we note that in Theorem 8 the $L^{p}$ condition on $\pi$ could without difficulty be replaced with an $L^{2}$ condition on $\tau$. Finally, we observe that neither of the conditions is actually as unnatural as it may first seem. They are both satisfied in a great many cases, including all connected semi-simple Lie groups with finite centre (see Cowling, [1] and Howe, [4]). 
EXAMPLE 1. Let $G$ be the circle group; let $x_{n}$ be the character $e^{\text {in } \theta}$. Then $X_{1}, X_{-1}$ are the only faithful irreducible representations (though of course there are many other faithful representations), and if, for example, we let $\pi=x_{1}$, then $\bar{\pi}=x_{-1}$, and $\pi_{m, n}=x_{m-n}$. Every $x_{k}$ equals some $\pi_{m, n}$ (in fact infinitely many).

EXAMPLE 2. Let $G=S L_{2}(R)$, or $P_{2 S L}(R)$. Then the results of Repka, [6] show that if $\pi$ is any non-trivial irreducible unitary representation, then the regular representation is contained in $\underset{m, n \leq 3}{\oplus^{\prime}} \pi_{m, n}$. This example also shows that $\sigma=\oplus^{\prime} \pi_{m, n}$ may contain subrepresentations which are not contained in the regular representation (this happens if $\pi$ is some "complementary series" representations).

\section{REFERENCES}

1. Cowling, M. Sur l'Algèbre de Fourier-Stieltjes d'un Groupe Semi-simple, preprint (1976).

2. Curtis, C.W. and Reiner, I. Representation Theory of Finite Groups and Associative Algebras, Interscience (1966).

3. Gelbart, S. Holomorphic Discrete Series for the Real Symplectic Group, Inv. Math. 19 (1973) 49-58.

4. Howe, R. On the Asymptotic Behaviour of Matrix Coefficients, preprint (1975).

5. Howe, R. Invariant Theory and Duality for Classical Groups over Finite Fields, preprint (1976).

6. Repka, J. Tensor Products of Unitary Representations of $\mathrm{SL}_{2}(\mathrm{R})$, Bull. Amer. Math. Soc. 82 (1976) 930-932; also to appear, Amer. J. Math.

7. Weil, A. Sur Certains Groupes d'Opérateurs Unitaires, Acta Math. 111 (1964) 143-211. 
KEY WORDS AND PHRASES. Locally compact group, compact group, unitary representation, tensor product, regular representation, Weil representation.

AMS (MOS) SUBJECT CLASSIFICATION (1970) CODES. 22010, $22 E 45$. 


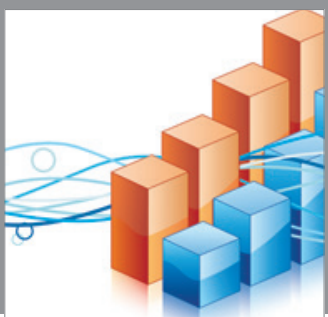

Advances in

Operations Research

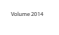

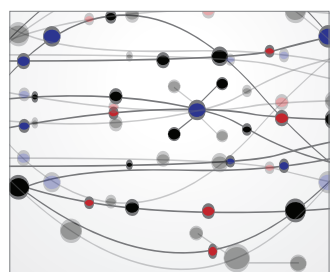

\section{The Scientific} World Journal
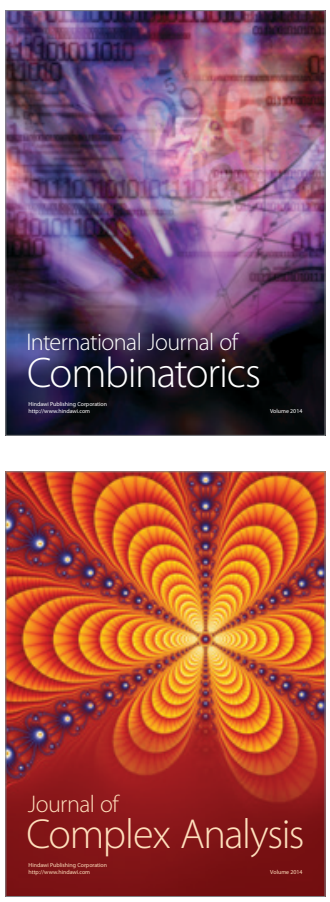

International Journal of

Mathematics and

Mathematical

Sciences
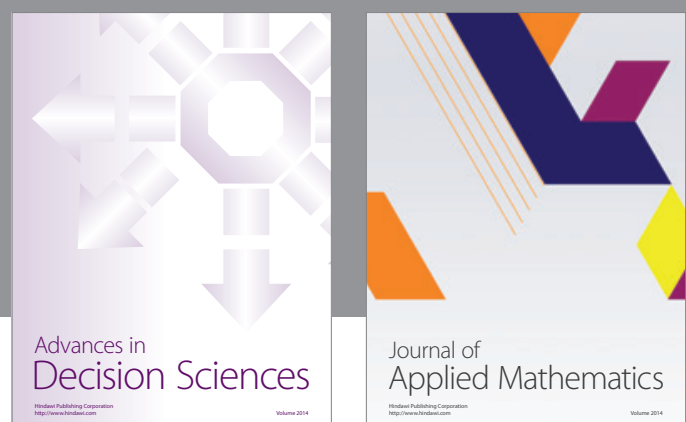

Journal of

Applied Mathematics
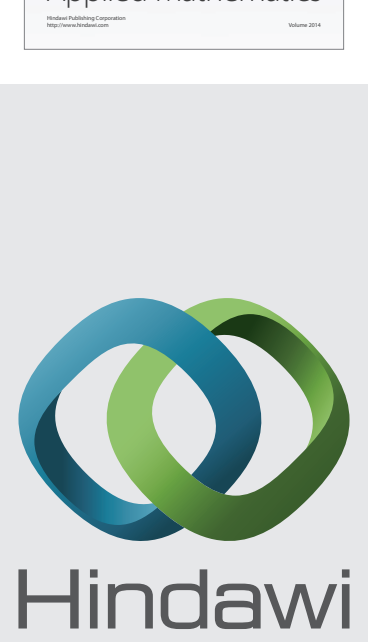

Submit your manuscripts at http://www.hindawi.com
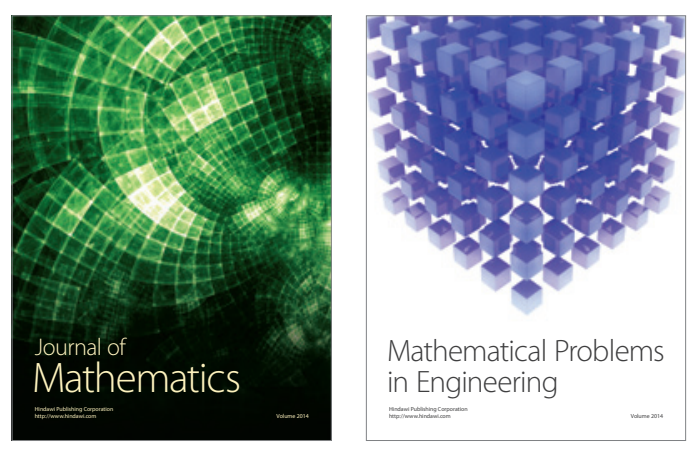

Mathematical Problems in Engineering
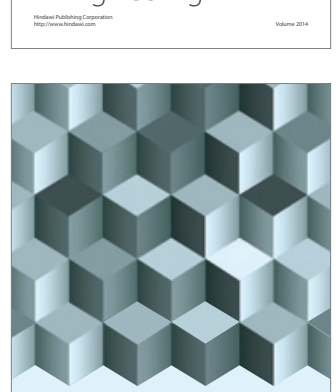

Journal of

Function Spaces
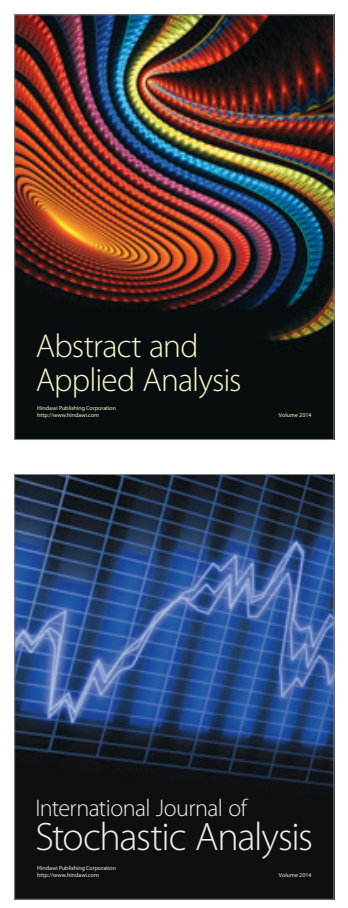

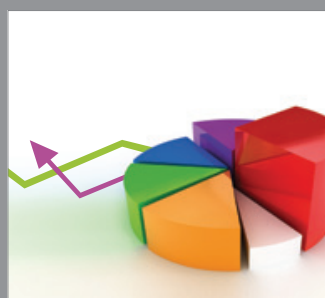

ournal of

Probability and Statistics

Promensencen
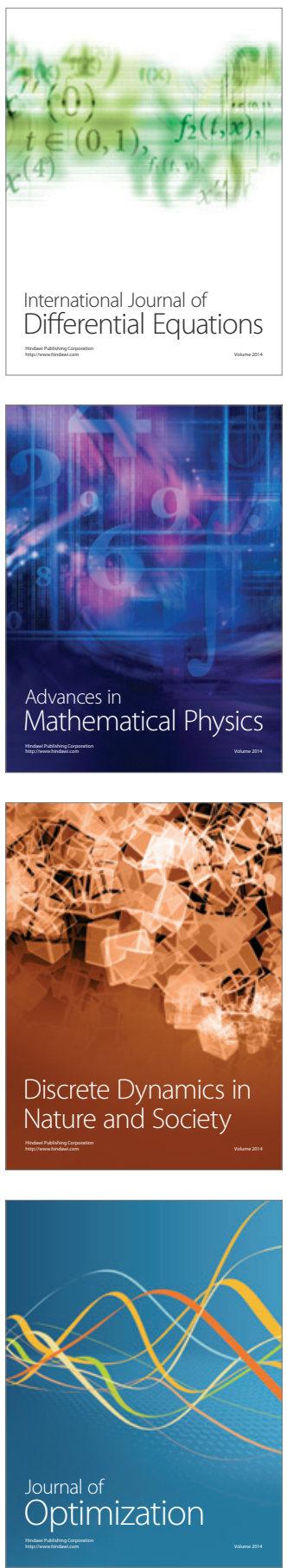